\title{
Lymphovascular tumor embolus might be a risk factor for peritoneal metastasis after laparoscopic hepatectomy for hepatocellular carcinoma
}

\author{
Zhiying Yang, Xiaolei Liu, Haidong Tan, Liguo Liu \\ Department of Hepatobiliary Surgery, China-Japan Friendship Hospital, Beijing 100029, China \\ Correspondence to: Zhiying Yang, MD. Department of Hepatobiliary Surgery, China-Japan Friendship Hospital, 2 East Yinghuayuan Street, Hepingli, \\ Beijing 100029, China. Email: yangzhy@aliyun.com.
}

Submitted May 30, 2018. Accepted for publication Jul 24, 2018.

doi: 10.21037/hbsn.2018.07.04

View this article at: http://dx.doi.org/10.21037/hbsn.2018.07.04

Laparoscopic hepatectomy has been applied to treat hepatocellular carcinoma (HCC) (1-7). Previous reports show that laparoscopic procedure is as safe as the open procedure, and the long-term results are also inspiring. However, there are still doubts about whether laparoscopic procedure would increase the possibility of peritoneal metastasis and what the risk factors may be. This issue has not been found or mentioned in these recent reports. However, we here present two patients with HCC who developed peritoneal metastasis after laparoscopic hepatectomy. Risk factors for peritoneal metastasis after laparoscopic hepatectomy merits further study.

From Jan 2013 to Jan 2017, 123 patients received laparoscopic hepatectomy in our center, including 25 HCC patients associated with liver cirrhosis (19 male and 6 female patients). The average age was 50 years old (range from 34 to 81 years). Based on their Child-Pugh scores, 24 patients were placed in class A and one patient was in class B. According to preoperative imaging, no peritoneal metastasis was found for all patients. Surgical procedures included 12 cases with left lateral sectionectomy, 3 cases with right hemihepatectomy, 5 cases left hemihepatectomy, and 5 cases with sectionectomy. No tumor rupture occurred during the procedure for all patients. Here, 21 patients had single lesion and 4 patients had multiple lesions. The average period of follow-up was 13 months (range, 5-41 months). The surgical margin was $>1 \mathrm{~cm}$ in 13 patients, $0.5-1 \mathrm{~cm}$ in 6 patients and $<0.5 \mathrm{~cm}$ in 6 patients. Postoperative pathology showed that lymphovascular tumor embolus was found in seven patients $(28 \%, 7 / 25)$. Two patients $(8 \%, 2 / 25)$ had peritoneal metastasis postoperatively and both of them had lymphovascular tumor embolus. The incidence of peritoneal metastasis for patients with lymphovascular tumor embolus was $28.6 \%(2 / 7)$. One patient with $7 \mathrm{~cm} \mathrm{HCC}$ in the right liver received laparoscopic right hemihepatectomy and the surgical margin was $0.5 \mathrm{~cm}$. Peritoneal metastasis occurred 4 months after the surgery. The other patient with $3 \mathrm{~cm}$ HCC of segment VI underwent laparoscopic multisectionectomy and the surgical margin was $2 \mathrm{~cm}$. Peritoneal metastasis occurred 3 months after the surgery.

Here, 82 HCC patients received open hepatectomy for HCC during the same period and 26 patients $(31.7 \%$, 26/82) had lymphovascular tumor embolus. The average period of follow-up was 15 months (range, 4-48 months) and no peritoneal metastasis occurred. The incidence of peritoneal metastasis showed no significant difference between open and laparoscopic procedures for HCC with cirrhosis $\left(0 \%\right.$ vs. $\left.8 \%, \chi^{2}=3.03, \mathrm{P}>0.05\right)$. However, it was significantly different for patients with lymphovascular tumor embolus ( $0 \%$ vs. $28.6 \%, \mathrm{P}=0.04)$. No peritoneal metastasis occurred in patients without lymphovascular tumor embolus regardless of open and laparoscopic procedure. Patients with lymphovascular tumor embolus had a higher incidence of peritoneal metastasis.

There are many causes for peritoneal metastasis of HCC patients who received laparoscopic procedure, such as tumor rupture during the surgery or inexperience of laparoscopic skills. The risk factors were still undetermined. Due to the limited number of patients in laparoscopic hepatectomy, it's not appropriate to draw a convincing conclusion. However, the phenomenon we found remind us the possibility that lymphovascular tumor embolus may be 
a risk factor of peritoneal metastasis for HCC patients who received laparoscopic procedure and further investigation would be needed.

\section{Acknowledgements}

None.

\section{Footnote}

Conflicts of Interest: The authors have no conflicts of interest to declare.

\section{References}

1. Han HS, Shehta A, Ahn S, et al. Laparoscopic versus open liver resection for hepatocellular carcinoma: Casematched study with propensity score matching. J Hepatol 2015;63:643-50

2. Sposito C, Battiston C, Facciorusso A, et al. Propensity score analysis of outcomes following laparoscopic or open liver resection for hepatocellular carcinoma. Br J Surg

Cite this article as: Yang Z, Liu X, Tan H, Liu L. Lymphovascular tumor embolus might be a risk factor for peritoneal metastasis after laparoscopic hepatectomy for hepatocellular carcinoma. HepatoBiliary Surg Nutr 2018;7(6):503-504. doi: 10.21037/hbsn.2018.07.04
2016;103:871-80.

3. Xiang L, Li J, Chen J, et al. Prospective cohort study of laparoscopic and open hepatectomy for hepatocellular carcinoma. Br J Surg 2016;103:1895-901.

4. Lee W, Han HS, Yoon YS, et al. Comparison of laparoscopic liver resection for hepatocellular carcinoma located in the posterosuperior segments or anterolateral segments: A case-matched analysis. Surgery 2016;160:1219-26.

5. Cheung TT, Dai WC, Tsang SH, et al. Pure Laparoscopic Hepatectomy Versus Open Hepatectomy for Hepatocellular Carcinoma in 110 Patients with Liver Cirrhosis. Ann Surg 2016;264:612-20.

6. Yoon YI, Kim KH, Kang SH, et al. Pure Laparoscopic Versus Open Right Hepatectomy for Hepatocellular Carcinoma in Patients With Cirrhosis A Propensity Score Matched Analysis. Ann Surg 2017;265:856-63.

7. Xu HW, Liu F, Li HY, et al. Outcomes following laparoscopic versus open major hepatectomy for hepatocellular carcinoma in patients with cirrhosis: a propensity score-matched analysis. Surg Endosc 2018;32:712-9. 